\title{
Un rôle possible pour l'EMDR : le traitement des traumas chez les patients psychotiques. Quatre vignettes cliniques
}

\author{
Richard Laugharne \\ University of Exeter Medical School, Cornwall, Royaume-Uni \\ Deborah Marshall \\ Cornwall Partnership NHS Trust, Cornwall, Royaume-Uni
}

Jon Laugharne

University of Western Australia, Perth, Australie

Alan Hassard

Plymouth Hospitals NHS Trust, Plymouth, Royaume-Uni

\begin{abstract}
Beaucoup de patients souffrant de psychose fonctionnelle ont connu un passé traumatique et présentent des symptômes d'ESPT (état de stress post-traumatique); certains peuvent aussi être traumatisés par leurs propres symptômes psychotiques. Nous présentons ici une série de vignettes cliniques décrivant le traitement EMDR (désensibilisation et retraitement par les mouvements oculaires) de quatre patients souffrant de psychose fonctionnelle et ayant tous un passé de traumatisation sévère. Après le traitement, tous les patients ont présenté une réduction de leurs symptômes d'ESPT et ont rapporté également une amélioration de leur qualité de vie. Le suivi, entre trois et six ans plus tard, a montré que les effets du traitement s'étaient maintenus, avec des changements manifestes : élimination des éléments délirants d'origine traumatique, réduction de l'anxiété et de la dépression, réduction du nombre d'hospitalisations, et amélioration globale de la qualité de vie des sujets. Comme les patients psychotiques ont plus fréquemment que les autres un passé de traumatismes et des symptômes d'ESPT, et que le trauma peut être une composante étiologique du déclenchement d'une psychose, il nous paraît nécessaire d'étudier et d'explorer l'EMDR en tant qu'opportunité de traitement.
\end{abstract}

Mots-clés : psychose ; trauma ; EMDR (désensibilisation et retraitement par les mouvements oculaires) ; psychothérapie ; résultat de traitement.

'EMDR (désensibilisation et retraitement par les mouvements oculaires) est une forme reconnue de traitement psychologique de l'état de stress post-traumatique (ESPT). Il existe une base importante de preuves scientifiques de son efficacité sur l'ESPT (Bisson \& Andrew, 2007), et l'EMDR est une approche thérapeutique recommandée pour le traitement de ce trouble par nombre de directives internationales, par exemple celles du United Kingdom National Institute for Health and Clinical Excellence (NICE, 2005).
Il est de mieux en mieux établi que le trauma joue un rôle important dans les psychoses. Les études montrent que plus de $50 \%$ des patients souffrant de psychoses fonctionnelles ont vécu des traumas dans leur enfance, proportion significativement plus élevée que celle de la population normale (Read, Van Os, Morrison \& Ross, 2005). Il y a un taux d'abus sexuels infantiles plus élevé chez les patients psychotiques que dans la population générale, et certaines études suggèrent que ce fait a une importance causale dans l'étiologie des psychoses (Bebbington et coll.,

This article originally appeared as Laugharne, R., Marshall, D., Laugharne, J. \& Hassard, A. (2014). A Role for EMDR in the Treatment of Patients Suffering From a Psychosis: Four Vignettes. Journal of EMDR Practice and Research, 8(1), 19-24. Translated by François Mousnier-Lompré. 
2011 ; Schäfer \& Fisher, 2011). De plus, la prévalence de l'ESPT est significativement plus élevée chez les patients psychotiques (Mueser et coll., 2004), et ceuxci souffrent souvent de symptômes d'ESPT résultant du traumatisme des symptômes psychotiques euxmêmes (Frame \& Morrison, 2000).

Il se peut que des patients psychotiques tirent profit de l'EMDR en se trouvant soulagés de symptômes d'ESPT provenant de traumatismes passés, et éventuellement des conséquences d'une réaction traumatique face à leurs propres symptômes. Une recherche a montré un bénéfice du travail EMDR sur des patients souffrant d'une forme particulière de psychose : quatre patients souffrant d'un syndrome de référence olfactive (SRO), syndrome dans lequel les personnes croient qu'elles émettent une odeur nauséabonde, et qui est un trouble considéré comme hallucinatoire par le Diagnostic and Statistical Manual of Mental Disorders ( quatrième édition ; DSM-IV), se sont pleinement rétablis grâce au traitement EMDR des événements qui avaient déclenché la pathologie (McGoldrick, Begum \& Brown, 2008). Une étude coréenne a traité des patients en phase schizophrénique aiguë, soit par l'EMDR, soit en relaxation musculaire, soit avec leur forme de thérapie habituelle (Kim et coll., 2010). Les patients continuaient de recevoir leur pharmacothérapie usuelle ; on les avait répartis au hasard, sans référence à des symptômes d'ESPT. Les trois groupes de patients ont présenté une amélioration similaire de leurs symptômes, même si, statistiquement, en raison du faible nombre des sujets (45 patients), on n'aurait pu détecter qu'un grand effet de taille.

Une étude pilote non contrôlée, aux Pays-Bas, a utilisé l'EMDR auprès de vingt-sept patients présentant des troubles du spectre schizophrénique et des troubles psychotiques non spécifiés, avec un ESPT comorbide. Après six séances d'EMDR, ces patients ont ressenti des améliorations importantes sur le plan des symptômes dépressifs, des hallucinations auditives et du délire, ainsi que de leurs symptômes d'ESPT (Van den Berg \& Van der Gaag, 2012). Dans une autre étude néerlandaise récente portant sur dix patients psychotiques ayant un ESPT comorbide, cinq ont été traités par exposition prolongée et cinq en EMDR (De Bont, van Minnen \& De Jongh, 2013). Les deux groupes ont montré des améliorations importantes de leurs symptômes d'ESPT, sans effets négatifs de la thérapie.

Dans le présent article, nous présenterons les vignettes de quatre patients ayant un diagnostic bien établi de psychose, à qui on a administré de l'EMDR pour des symptômes d'ESPT. Ils ont tiré profit du traitement, et nous pensons que leur qualité de vie s'en est trouvée améliorée. Ces cas cliniques faisaient partie de la file active habituelle des services britanniques de santé (NHS), et nous n'avons pas utilisé d'échelle de recherche, d'échelle de diagnostic, ni d'échelle d'évaluation d'étude. Nous avons cherché à mettre en lumière l'utilité thérapeutique de l'EMDR dans cette population.

\section{Cas cliniques :}

\section{Vignette $1: A D$}

À l'époque où nous avons fait de l'EMDR avec lui, $\mathrm{AD}$ était un jeune homme de 28 ans avec un diagnostic de schizophrénie et un passé de symptômes anxieux et dépressifs importants. Il présentait également une dépendance aux opiacés. À 19 ans, il avait eu un grave accident de la route dans lequel deux de ses amis avaient trouvé la mort, l'un d'eux s'étant retrouvé couché sur lui au cours de l'accident; $\mathrm{AD}$, quant à lui, avait eu de graves blessures à la tête, qui avaient mis des mois à guérir. Il avait commencé à présenter des symptômes de schizophrénie à partir de l'âge de 20 ans, avec un délire de persécution et des hallucinations. Les tests neuropsychologiques ne montraient pas de déficiences cognitives résiduelles qui auraient clairement pu être responsables de ses symptômes psychotiques.

Quand nous l'avons vu, à l'âge de vingt-huit ans, pour faire de l'EMDR avec lui, $\mathrm{AD}$ avait régulièrement des cauchemars et des flash-backs de l'accident, mais ne présentait ni délire ni hallucinations. Il prenait des antipsychotiques et des antidépresseurs ; sa psychose était bien contrôlée, mais l'angoisse était pour lui un problème important et il avait tendance à ne pas prendre soin de lui-même et à présenter des symptômes négatifs. Il était sous méthadone par rapport à son héroïnomanie; il n'achetait pas de drogue dans la rue. Il avait été évalué par le psychiatre qui le suivait en ambulatoire et qui était également son thérapeute EMDR (le premier auteur, RL). Le psychiatre assurait le suivi d'AD depuis deux ans, y compris les hospitalisations, et la relation thérapeutique était donc bien établie.

Nous avons fait avec AD trois séances d'EMDR sur son souvenir de l'accident de la route. On a utilisé le protocole standard d'EMDR en huit étapes (Shapiro, 2001), tel qu'il est enseigné par l'institut EMDR britannique : recueil de l'histoire, préparation, avec la création d'un lieu sûr, évaluation du souvenir (identification d'une image de l'incident, émotion et sensation corporelle associées, cognition négative et cognition positive), désensibilisation, installation, scanner 
corporel et clôture. La cognition négative était : «Je suis responsable de l'accident ». La cognition positive était : "Je peux aller bien ». Chaque fois, on a utilisé des séquences de vingt-quatre mouvements oculaires bilatéraux, en suivant les doigts du thérapeute. On a fait ces mouvements oculaires au cours des étapes de désensibilisation et d'installation. Ce processus a été suivi à chaque séance, il n'a entraîné l'apparition d'aucun symptôme psychotique, et le patient n'a pas subi de stress excessif.

Suite à ces séances, la perturbation d'AD en se remémorant l'incident s'est trouvée considérablement réduite. Les cauchemars et les flash-backs se sont améliorés de façon importante. Il en était de même de sa dépression et de son anxiété, jusque-là chroniques, et pour lui, l'amélioration de son sommeil, suite à la réduction du nombre des cauchemars, a été particulièrement bénéfique.

Même si l'abus d'opiacés était encore par moments un problème, le fonctionnement général du patient s'est amélioré graduellement, car il lui a été possible de vivre chez lui, sans retours réguliers à l'hôpital. Depuis peu, il envisage à nouveau de conduire. Au cours de ce travail en EMDR, il n'a eu ni délire ni hallucinations, et a poursuivi son traitement antipsychotique. Il a été accompagné par une équipe ambulatoire expérimentée, qui a rapporté une amélioration importante de son fonctionnement. Cette amélioration dure maintenant depuis six ans, sans anxiété ni dépression. Les visites au psychiatre ont été ramenées à une par mois.

\section{Vignette 2 : BK}

BK, un homme de 32 ans, souffrait d'un trouble bipolaire. Il avait un passé d'épisodes maniaques depuis une dizaine d'années, au cours desquels il était persuadé qu'on le gazait et que les soignants allaient le tuer. Il avait connu, dans le passé, des hospitalisations d'office. Au moment de l'évaluation, BK, contre l'avis des soignants, n'avait plus de traitement médicamenteux du fait de son refus d'en prendre, et il était retourné chez lui. Il ne présentait ni hallucinations ni délire, et il était euthymique. Il a été évalué par le psychiatre qui s'était chargé de son suivi à sa sortie de l'hôpital, et qui allait être aussi son thérapeute EMDR (le premier auteur, RL) ; le patient ne connaissait pas le thérapeute.

Lors de cette évaluation, BK révéla qu'à l'âge de six ans, il avait dû subir une chirurgie orthopédique lourde. Lors d'une intervention, en salle d'opération, l'anesthésiste ne parvint pas à poser une voie veineuse ; en conséquence, on dut endormir l'enfant à l'aide d'un gaz anesthésiant, mais il crut que les médecins essayaient de lui faire du mal, car on le maintint de force pour lui administrer le gaz. Ces souvenirs provoquaient une grande détresse chez BK, et ils étaient associés à des flash-backs et à des cauchemars. Il est frappant de constater que certaines idées délirantes de BK reflétaient ces souvenirs traumatiques, puisqu'il croyait, en unité de soins intensifs psychiatriques, que l'équipe cherchait à le tuer, entre autres en envoyant du gaz empoisonné par les grilles d'aérations de la chambre d'isolement.

On lui proposa l'EMDR. BK, souhaitant être aidé sur le plan de ses souvenirs traumatiques, donna son accord. Il eut deux séances d'EMDR, la première centrée sur les événements qu'il avait vécus à six ans, la seconde sur son séjour en isolement, où il avait eu ces idées persécutoires concernant le personnel hospitalier. Comme pour $\mathrm{AD}$, nous avons suivi scrupuleusement le protocole standardisé enseigné au Royaume-Uni par l'Institut d'EMDR américain : établissement d'un lieu sûr, image de l'incident, cognition négative et cognition positive. Sa cognition négative était : " Je suis impuissant et tout seul». La cognition positive était : «Je suis en mesure de contrôler la situation ». Nous avons fait les mouvements oculaires pour la désensibilisation avant les mouvements oculaires du retraitement.

Le traitement EMDR a produit une réduction notable du niveau de perturbation lié à ces souvenirs. Les principaux changements ont été une raréfaction des cauchemars et des flash-backs et une détresse beaucoup moins importante lorsqu'il se rappelait son opération à six ans, ce qui a amélioré significativement sa qualité de vie. BK a eu ensuite un autre épisode maniaque, nécessitant une hospitalisation, mais il a rapporté à sa sortie que les cauchemars et les flash-backs n'étaient pas revenus, même lorsqu'il était en crise à l'hôpital. Il pense qu'une désintoxication du mercure, avec le retrait d'amalgames dentaires, a contribué à cette amélioration. Il a été d'accord pour prendre volontairement des psychotropes en ambulatoire, et il est suivi par les services psychiatriques de secteur. Cinq ans plus tard, il va bien, même s'il a connu une réhospitalisation entre-temps.

\section{Vignette $3: R L$}

RL est un homme de vingt-huit ans, avec un passé de toxicomanie, de délire de persécution (même à des périodes où il ne se droguait pas), de pensées obsessionnelles intrusives, de symptômes anxieux et de symptômes dépressifs. Son diagnostic primaire est la schizophrénie. Il est sous traitement antipsychotique, sous antidépresseurs, et prend une benzodiazépine. 
RL avait des cauchemars qui se rapportaient à un incident auquel il n'avait pourtant pas assisté : un de ses associés en avait poignardé un autre et l'avait tué. Il avait des cauchemars autour de cet incident environ cinq nuits par semaine. Il y voyait toujours son associé, debout, le couteau à la main, au-dessus du corps de l'autre. Après ces cauchemars, il se sentait agité et ses " pensées paranoïdes » s'aggravaient quand il avait l'impression que les autres étaient contre lui ou parlaient de lui. Il n'avait pas de symptôme psychotique permanent à cette période, car le traitement médicamenteux semblait les contrôler. Le psychiatre qui le suivait était aussi son thérapeute EMDR (le premier auteur, RL) ; il le suivait depuis deux ans. RL a été évalué dans le service de psychiatrie de secteur.

Il a reçu trois séances d'EMDR. Le protocole manualisé en huit étapes, tel qu'enseigné au Royaume-Uni par l'Institut d'EMDR, a été là aussi suivi à la lettre. Nous nous sommes centrés sur le contenu de ses cauchemars et les opinions négatives de lui-même qui s'y trouvaient associées. La cognition négative était : "Je risque tout le temps de me mettre dans des ennuis». La cognition positive était : "Je peux rester à l'écart des ennuis ". L'EMDR a pu avancer sans apparition de symptôme psychotique ; RL a supporté les séances sans difficulté. Nous avons utilisé des séquences de vingt-quatre mouvements oculaires tant dans la phase de désensibilisation que dans la phase d'installation du traitement. Après trois séances, la détresse associée aux images des cauchemars avait régressé. Il rapporta deux mois plus tard qu'il n'avait plus qu'un cauchemar par mois ; en conséquence, son agitation et ses idées persécutoires avaient diminué.

Aujourd'hui, trois ans après, ses difficultés n'ont aucunement disparu, car il se débat encore avec des pensées intrusives et des problèmes de contrôle de la colère. Cependant, son fonctionnement global s'est amélioré. Il n'habite plus avec sa mère, il vit de façon autonome et ne se drogue plus. Il prend un traitement antipsychotique et ne souffre plus, de façon générale, de délire ou d'hallucinations. Il a eu une rechute, avec une réhospitalisation, suite à un arrêt de son antipsychotique - le traitement a ensuite été repris. Lorsqu'il est sous médication, il vit de façon plus indépendante ; il prend régulièrement en charge son jeune fils, qui vit avec son ex-compagne.

\section{Vignette 4 : NP}

Cet homme a été adressé à l'âge de vingt-deux ans à notre équipe d'intervention précoce auprès des patients psychotiques, avec un diagnostic de psychose non autrement spécifiée. Son principal souci, lorsqu'il était en crise, était que la police cherchait à l'arrêter. Cette croyance provenait du souvenir d'un incident traumatique avec la police, trois ans plus tôt : les policiers avaient cassé la vitre de la voiture dans laquelle il se trouvait et l'en avaient extrait par l'ouverture ainsi pratiquée. On l'avait laissé partir, avec un avertissement pour son rôle dans l'incident. Il en avait gardé des souvenirs traumatiques et avait apparemment fait un épisode psychotique en rapport direct avec cet incident.

Au cours de cet épisode psychotique, il expliqua que des policiers, la nuit, tournaient autour de chez lui et parlaient de lui. Des voitures de police, selon lui, se tenaient devant chez lui, avec leurs gyrophares allumés et leurs sirènes hurlantes. Il était extrêmement anxieux la plupart du temps, sortait rarement de chez lui et avait inversé son rythme de sommeil, car, de peur d'être arrêté, il lui fallait rester hypervigilant la nuit.

Il a été traité avec divers antipsychotiques : rispéridone, trifluopérazine, olanzapine ; on lui a également prescrit de temps en temps du diazépam, du valproate de sodium, de la venlaxafine et du citalopram. NP a donné son accord pour avoir des séances d'EMDR avec son infirmière psychiatrique de secteur (le second auteur, DM). Au moment où l'EMDR a été engagée, il avait un traitement antipsychotique et n'était pas en phase délirante ou hallucinatoire aiguë. Il a choisi comme cognition négative : "Je suis impuissant » et comme cognition positive : «Je peux surmonter ça et continuer ma vie ». Il décrivait ses émotions comme " panique et frayeur "; physiquement, ses symptômes se trouvaient localisés dans ses mains, ses jambes, avec une impression de gorge serrée et d'étouffement. Il a participé seulement à deux séances d'EMDR ; au cours de celles-ci, il a revécu son expérience et a pu la fractionner en plusieurs étapes. Il a passé deux fois le souvenir entier de l'incident, et a pu se rappeler plusieurs détails positifs qu'il n'avait pu retrouver auparavant. Il s'est ainsi souvenu d'un policier qui s'était positionné en sa faveur et l'avait apparemment défendu en écartant de force un autre policier, alors qu'on maitrisait physiquement NP. À la fin de la seconde séance, il a pu dire qu'il ne ressentait plus qu'une légère anxiété en repensant à son arrestation.

Par la suite, il s'est engagé plus complètement dans le travail avec sa thérapeute. Il n'avait plus d'attaques de panique, et, point important, il a pu se rendre dans un commissariat pour parler de sa peur d'être toujours recherché. Les policiers ont alors pu le rassurer. Il avait le sentiment que l'EMDR avait réduit la force du système de croyances et l'extrême paranoïa qu'il 
connaissait dans ses épisodes psychotiques. Grâce à la thérapie, il était moins perturbé quand il pensait à cet incident, ce qui lui avait permis de se rendre au commissariat de police.

Au cours des quatre années suivant le traitement EMDR, NP a eu d'autres épisodes psychotiques, mais son délire ne portait plus sur une terreur de la police. Il continue de prendre son traitement antipsychotique. Il n'avait plus affaire depuis quelque temps aux services psychiatriques, mais il a présenté à nouveau des symptômes psychotiques, et on explore actuellement chez lui une éventuelle épilepsie temporale.

\section{Discussion}

Ces vignettes présentent des patients souffrant de graves troubles psychotiques fonctionnels, et dont la vie a été affectée par des traumas de nature diverse. Ils ont tous reçu de l'EMDR pour aborder les symptômes d'ESPT associés à ces traumas, ESPT comorbide à leurs troubles psychotiques. Tous ont tiré un bénéfice de leur traitement EMDR : ils l'ont rapporté eux-mêmes, et les thérapeutes l'ont observé.

Pour AD, BK et RL, le thérapeute EMDR était un psychiatre (le premier auteur, RL) également responsable du traitement médicamenteux et de la prise en charge globale de ces patients ; tous trois recevaient également des soins des infirmiers d'une équipe de psychiatrie de secteur. Pour NP, la thérapeute (le second auteur, DM) était une infirmière psychiatrique de secteur travaillant au sein d'une Équipe d'intervention précoce. Ainsi, pour tous les patients, s'il y avait eu un éventuel effet négatif de l'EMDR, le thérapeute et la thérapie étaient partie intégrante de l'ensemble de leurs soins. Les deux thérapeutes ont été formés au Royaume-Uni par l'Institut EMDR américain. Aucun des patients n'était en phase psychotique aiguë au moment de l'EMDR, et aucun n'était hospitalisé. Ils n'ont pas eu besoin d'une longue préparation à l'EMDR, car les deux thérapeutes EMDR étaient un psychiatre et une infirmière de secteur qui les connaissaient bien et qui faisaient partie de leur environnement de soins habituel ; le fait qu'ils aient eu ces rôles classiques, tout en étant thérapeutes EMDR, peut aussi avoir donné confiance à ces derniers dans le fait de pratiquer l'EMDR sans une longue préparation préalable.

Ces patients ont fait de l'EMDR dans le cadre habituel des soins du service britannique de santé publique. Nos vignettes ont pour limite l'absence d'échelles de diagnostic et de symptômes comparables à celles qu'on utilise dans les études plus systématiques, et elles sont donc purement descriptives. Si nous ne pouvons prétendre avoir démontré l'efficacité de l'EMDR chez les patients psychotiques, nos vignettes mettent en lumière et illustrent l'utilité de l'EMDR auprès de cette population clinique. Un ESPT n'avait pas été systématiquement diagnostiqué chez nos patients, et nous ne pouvons donc pas affirmer qu'ils en remplissaient les critères ; cependant, les deux thérapeutes ont identifié d'importants symptômes (cauchemars, flashbacks et/ ou hypervigilance), et le traitement psychothérapique a eu lieu dans un service de soins « réel».

Nous avons fait de l'EMDR avec nos patients dans le cadre d'un ensemble global de soins, et nous ne pouvons affirmer une claire relation de cause à effet entre des améliorations constatées dans leur vie et l'EMDR. Mais nous pouvons faire du moins l'hypothèse, à partir de cette expérience clinique, qu'auprès de patients psychotiques ayant vécu des traumas, l'EMDR peut apporter des améliorations, à la fois subjectives et objectives, sur le plan de leur fonctionnement et de leur qualité de vie. Le traitement EMDR n'a entraîné aucune aggravation d'un symptôme psychotique au cours des séances. Il y a eu récemment un essai clinique d'EMDR auprès de patients psychotiques, sans référence à des symptômes d'ESPT et pendant une période aiguë de la maladie (Kim et coll., 2010). Une autre étude-pilote a effectivement constaté les résultats positifs d'un travail EMDR auprès de patients souffrant à la fois de psychose et d'ESPT (Van den Berg \& Van der Gaag, 2012). Cette étude pilote a montré une amélioration des symptômes d'ESPT et de certains symptômes psychotiques comme les idées délirantes et les hallucinations auditives, mais pas de l'idéation paranoïde. Il est intéressant de noter que nous avons trouvé la preuve clinique d'une réduction des pensées paranoïdes chez deux de nos patients (RL et NP), suite au traitement EMDR. On a des preuves préliminaires de l'efficacité de la thérapie cognitivecomportementale par exposition (TCC), chez des patients présentant un ESPT et une schizophrénie et un trouble schizoaffectif comorbides (Frueh et coll., 2009). L'EMDR peut constituer une alternative pour des patients qui ne répondent pas à la TCC, ou ont du mal à s'y engager ou à se plier au travail personnel qui est partie intégrante de la TCC.

Nous avançons l'hypothèse que l'EMDR peut être une forme efficace de traitement des symptômes d'ESPT chez les patients psychotiques. Nous estimons qu'il vaut mieux la délivrer dans une phase plus stable de la maladie, quand les patients sont capables de comprendre et de se concentrer sur le processus. La vignette $2(\mathrm{BK})$ nous mène aussi à poser l'hypothèse qu'en abordant et en traitant les vécus traumatiques antérieurs des patients, ceux-ci se sentent écoutés et l'amélioration de la relation thérapeutique qui 
en découle peut les amener à une meilleure observance des traitements médicamenteux qui leur sont recommandés.

Nous pensons, suite à ces études, qu'il est nécessaire de mener des essais contrôlés de l'EMDR avec des patients psychotiques qui ont un ESPT comorbide, qui ont un passé traumatique sans symptôme d'ESPT, ou encore qui présentent des symptômes d'ESPT suite à leurs symptômes psychotiques : on ne peut en effet guère tirer de conclusions solides sur le plan de la politique de santé tant qu'on manque d'essais contrôlés évaluant l'efficacité de l'EMDR dans les psychoses. Cependant, à notre sens, l'EMDR est une option thérapeutique importante pour les patients psychotiques ayant des symptômes d'ESPT comorbide suite à des traumas. Le trauma étant plus fréquent dans ce groupe de patients que dans la population générale, cela ne doit pas être considéré comme un risque, mais comme une opportunité thérapeutique, pour autant que l'EMDR soit intégrée dans une offre de soins globale qui comprend aussi les nécessaires traitements fondés sur la preuve. Nous suggérons aux cliniciens qu'il serait avisé de rechercher chez tous les patients psychotiques des symptômes d'ESPT et de leur proposer, dans le cadre d'un plan de traitement global, des thérapies centrées sur les traumas.

\section{Consentement}

Les patients décrits ici ont lu cet article et ont donné leur consentement écrit pour la publication de leur cas. Leurs identités ont été anonymisées.

\section{Bibliographie}

Bebbington, P., Jonas, S., Kuipers, E., King, M., Cooper, C., Brugha, T.,..., Jenkins, R. (2011). Childhood sexual abuse and psychosis: Data from a cross sectional national psychiatric survey in England. British Journal of Psychiatry, 199, 29-37.

Bisson, J. \& Andrew, M. (2007). Psychological treatment of post-traumatic stress disorder. Cochrane Database Systemic Review, (3), CD003388.

De Bont, P. A., van Minnen, A. \& De Jongh, A. (2013). Prolonged exposure and EMDR for PTSD in patients with psychosis. A randomized multiple baseline controlled study. Behavior Therapy, 44, 717-730.

Frame, L. \& Morrison, A. (2001). Causes of posttraumatic stress disorder in psychotic patients. Archives of General Psychiatry, 58, 305-306.

Frueh, B. C., Grubaugh, A. L., Cusack, K. J., Kimble, M. O., Elhai, J. D. \& Knapp, R. G. (2009). Exposure based cognitive-behavioural treatment of PTSD in adults with schizophrenia or schizoaffective disorder: A pilot study. Journal of Anxiety Disorders, 23, 665-675.

Kim, D., Choi, J., Kim, S. H., Hoon, D., Park, S. C. \& Lee, S. H. (2010). A pilot study of brief eye movement desensitization and reprocessing (EMDR) for acute phase schizophrenia. Korean Journal of Biological Psychiatry, 17, 94-102.

McGoldrick, T., Begum, M. \& Brown, K. W. (2008). EMDR and olfactory reference syndrome: A case series. Journal of EMDR Practice and Research, 2, 63-68.

Mueser, K. T., Salyers, M. P., Rosenberg, S. D., Goodman, L. A., Essock, S. M., Osher, F. C., ..., Butterfield, M. I. (2004). Interpersonal trauma and post traumatic stress disorder in patients with severe mental illness. Schizophrenia Bulletin, 30, 45-57.

National Institute of Health and Clinical Excellence (NICE). (2005). Clinical practice guideline CG26: Posttraumatic stress disorder. Leicester, United Kingdom: Gaskell.

Read, J., Van Os, J., Morrison, A. P. \& Ross, C. A. (2005). Childhood trauma, psychosis and schizophrenia: A literature review with theoretical and clinical implications. Acta Psychiatrica Scandinavica, 112(5), 330-350.

Schäfer, I. \& Fisher, H. L. (2011). Childhood trauma and posttraumatic stress disorder in patients with psychosis: Clinical challenges and emerging treatments. Current Opinion in Psychiatry, 24, 514-518.

Shapiro, F. (2001). Eye movement desensitization and reprocessing: Basic principles, protocols and procedures (2nd ed.). New York, NY, Guilford Press.

Van den Berg, D. P., \& van der Gaag, M. (2012). Treating trauma in psychosis with EMDR: A pilot study. Journal of Behavior Therapy \& Experimental Psychiatry, 43, 664-671.

Merci d'adresser toute correspondance concernant cet article à : Docteur Richard Laugharne, Cornwall Partnership NHS Foundation Trust, Lodge Hill, Liskeard, Cornwall, PL14 4EN, Royaume-Uni. Courriel : r.laugharne@exeter .ac.uk ou richard.laugharne@pms.ac.uk 